\title{
ESTIMAÇÃO DA FUNÇÃO DE SENSIBILIDADE BASEADA EM EXPERIMENTO COM RELÉ EM MALHA FECHADA
}

\author{
Gustavo H. M. de Arruda * \\ arruda@dee.ufcg.edu.br
}

\author{
Péricles R. Barros* \\ prbarros@dee.ufcg.edu.br
}

* Laboratório de Instrumentação Eletrônica e Controle, Departamento de Engenharia Elétrica, Universidade Federal de Campina Grande, Caixa Postal 10105, 58109 - 970 Campina Grande, PB - BRASIL.

\begin{abstract}
A new technique for relay based closed loop transfer function estimation is presented. Given a closed loop system and a user selected transfer function, then a relay experiment is used to obtain the frequency corresponding to a selected transfer function magnitude. In this paper the Sensitivity Function is considered. This information can be used to evaluate closed loop properties and/or to redesign the controller.
\end{abstract}

KEYWORDS: Relay feedback, identification, sensitivity function

\section{RESUMO}

Uma nova técnica de estimação usando o relé em malha fechada é apresentada. Dado um sistema em malha fechada e uma função de transferência associada, um experimento com relé é utilizado para obter a freqüência na qual a função de transferência escolhida possui uma magnitude desejada. Neste artigo, será considerada a Função de Sensibilidade. Esta informação pode ser usada para se avaliar as propriedades do sistema em malha fechada e/ou reprojetar o controlador.

PALAVRAS-CHAVE: Relé, identificação de sistemas, função de sensibilidade

Artigo submetido em 20/12/00

1a. Revisão em 22/3/2002; 2a. Revisão em 31/10/2002;

3a. Revisão em $27 / 11 / 02$

Aceito sob recomendação do Ed. Assoc. Prof. José R. C. Piqueira

\section{INTRODUÇÃO}

O método do relé introduzido por Åström e Hägglund (1984) tem sido amplamente utilizado como ferramenta de estimação para sintonia on-line de controladores, devido à sua simplicidade. Na sua forma original, um relé em malha fechada é utilizado para estimar o ganho e a freqüência críticos de um processo, sob condições de operação bem definidas. A informação obtida do experimento pode ser utilizada para a sintonia de controladores PID, através do método da resposta em frequiência de Ziegler-Nichols, ou variações do mesmo, como os métodos de Ziegler-Nichols modificado, Chien-HronesReswick, Cohen-Coon e Tyreus-Luyben (ver em Tan et al. (1999) e Åström e Hägglund (1995)). A análise de uma malha com realimentação por relé é feita pelo método das funções descritivas, o que tem contribuído para tornar o experimento largamente aceito.

O método original tem sido aprimorado para a estimação de diferentes pontos da resposta em freqüência de um processo. Em trabalho recente (Wang, Hang e Bi, 1999), a resposta transitória durante o início de um único experimento com relé é utilizada para estimar vários pontos de freqüência simultaneamente. Um método alternativo, baseado no modelo de filtro de amostragem de freqüência, é utilizado em Wang, Desarmo e Cluett (1999) para estimar pontos da resposta em freqüência a partir de dados de entrada e saída do processo. Em Schei (1992) o relé é utilizado na malha fechada de modo que um ciclo limite se desenvolve na freqüência em que o ângulo de fase da Função de Sensibilidade Complementar é 
igual a $-90^{\circ}$. Tal freqüência se encontra entre a freqüência de cruzamento do ganho ${ }^{1}$ e a freqüência crítica da Função de Malha do sistema. Em Schei (1994), um novo experimento com relé é proposto, em que o sistema em malha fechada oscila aproximadamente na freqüência de cruzamento do ganho. Em de Arruda e Barros (2000b), tal experimento é generalizado de modo que o ciclo limite é desenvolvido na freqüência em que a magnitude da Função de Malha assume um valor desejado.

A Função de Sensibilidade contém informações importantes sobre a malha fechada como, por exemplo, margens de estabilidade e rejeição a perturbações (Doyle et al., 1995). Avaliar experimentalmente a Função de Sensibilidade é importante para determinar se especificações de margens de estabilidade e rejeição a perturbações são satisfeitas. Tais informações também podem ser utilizadas no reprojeto de controladores. A moldagem espectral da Função de Sensibilidade (sensitivity shaping) é uma abordagem alternativa para o projeto de controladores, como apresentada em Doyle et al. (1995), Langer e Landau (1999) e Barros e Wittenmark (1997).

Este artigo é uma extensão da versão de de Arruda e Barros (2000a), onde é apresentada uma técnica de identificação no domínio da freqüência utilizando o relé na malha fechada. A técnica, aqui denominada procedimento básico, é utilizada na identificação da Função de Sensibilidade (seção 4) por meio de uma nova estrutura de realimentação descrita na seção 3. Não existe nenhuma outra técnica na literatura baseada em experimentos com relé para identificação da Função de Sensibilidade de conhecimento dos autores. Aspectos práticos, como amplitude do relé e estabilidade da malha fechada, são discutidos na seção 5. Finalmente, um exemplo de simulação e resultados experimentais com um sistema de controle de temperatura são mostrados nas seções 7 e 8 .

\section{NOTAÇÃO}

Considere o sistema em malha fechada mostrado na Fig. 1. A função de transferência do processo é dada por $G(s)$, e o controlador é dado por $C(s)$. A função de transferência de malha fechada da referência $y_{r}(t)$ para a saída $y(t)$ é dada por

$$
T(s)=\frac{Y(s)}{Y_{r}(s)}=\frac{C(s) G(s)}{1+C(s) G(s)} .
$$

Para esta configuração de malha fechada, $T(s)$ é também conhecida por Função de Sensibilidade Complementar, $L(s)=C(s) G(s)$ é a Função de Transferência

\footnotetext{
${ }^{1} \mathrm{~A}$ freqüência na qual o ganho da Função de Malha é unitário.
}

da Malha e

$$
S(s)=\frac{E(s)}{Y_{r}(s)}=\frac{1}{1+C(s) G(s)},
$$

é a Função de Sensibilidade.

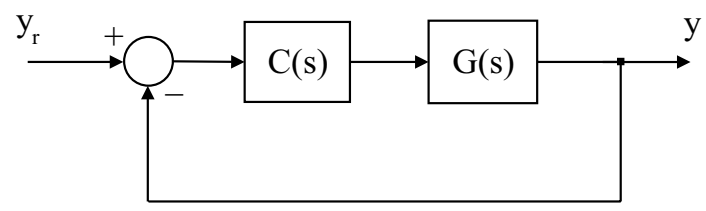

Figura 1: Sistema em Malha Fechada.

\section{O MÉTODO DO RELÉ}

No experimento original de Ziegler e Nichols (1942), são determinados os valores da freqüência e ganho críticos do processo ( $\omega_{u}$ e $K_{u}$, respectivamente), variando o ganho de um controlador proporcional, $C(s)=K$. Os parâmetros do controlador PID são obtidos a partir de uma tabela em função de $K_{u}$ e $\omega_{u}$. A principal desvantagem deste experimento está no fato de ter que levar o processo ao limite de estabilidade, a fim de determinar o ponto crítico. Além disso, não se tem controle sobre a amplitude da oscilação na saída do processo, de modo que o experimento geralmente é impraticável, por razões de segurança ou custo.

Åström e Hägglund (1984) propuseram uma forma alternativa para determinar $K_{u}$ e $\omega_{u}$ utilizando uma realimentação com relé. Considere o sistema em malha fechada da Fig. 2, em que o relé tem amplitude $d, y_{r}=0$ e o elemento linear $\alpha(s)=1$. Da análise por função descritiva mostra-se que, para várias classes de processos, o sistema exibe um ciclo limite aproximadamente no ponto em que

$$
N(a) G(j \omega)=-1
$$

com

$$
N(a)=\frac{4 d}{\pi a},
$$

sendo a função descritiva do relé. Assim, se $a$ é a amplitude de oscilação na saída do processo, a intersecção da curva de Nyquist do processo com a função descritiva do relé ocorre no ponto crítico do processo, de modo que

$$
G\left(j \omega_{u}\right)=-\frac{\pi a}{4 d} .
$$

Note agora que, neste caso, a amplitude de oscilação na saída do processo pode ser facilmente controlada a partir da amplitude $d$ do relé. 


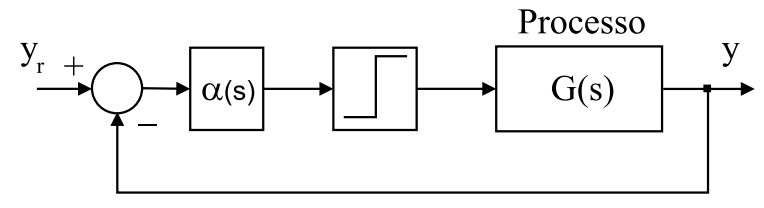

Figura 2: Processo com Realimentação por Relé.

Um segundo experimento pode ser obtido introduzindo um integrador no ramo direto mostrado na Fig. 2, i.e., $\alpha(s)=1 / s$. Neste caso,

$$
G\left(j \omega_{u}\right)=-j \frac{\pi a \omega_{o}}{4 d},
$$

de modo que a freqüência de oscilação $\omega_{o}$ é tal que o ângulo de fase do processo nesta freqüência é aproximadamente $-90^{\circ}$.

\section{UM NOVO EXPERIMENTO COM RELÉ EM MALHA FECHADA}

Considere o sistema em malha fechada com relé, apresentado na Fig. 3, em que a função de transferência $F(s)$ é estável. Da análise por função descritiva (Åström e Hägglund, 1995), mostra-se que o sistema em malha fechada está em ciclo limite com freqüência de oscilação tal que $\angle F(j \omega) \cong-90^{\circ}$. Se $F(s)=T(s)$, então obtém-se a estrutura apresentada em Schei (1992), e com $F(s)=2 T(s)-1$, obtém-se a estrutura apresentada em Schei (1994). Neste último, mostra-se que a freqüência de oscilação do ciclo limite é aproximadamente igual à freqüência de cruzamento do ganho. Este resultado é reproduzido a seguir para facilitar o entendimento do método.

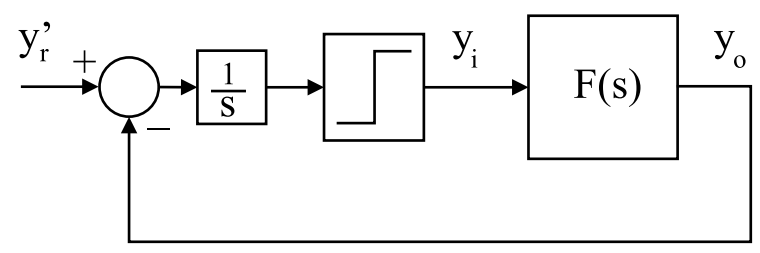

Figura 3: Diagrama em Blocos das Estruturas Apresentadas em Schei (1992) e Schei (1994).

Proposição 1 Considere o sistema em malha fechada com relé mostrado na Fig. 3, onde $F(s)=2 T(s)-1$, $T(s)$ dado pela Eq. (1), e assuma que existe um ciclo limite estável. Então o sistema em malha fechada oscila na freqüência $\omega_{l c}$ para a qual

$$
\left|L\left(j \omega_{l c}\right)\right|=\left|C\left(j \omega_{l c}\right) G\left(j \omega_{l c}\right)\right| \cong 1 .
$$

Prova: Ver em Schei (1994).

A realimentação que implementa $F(s)=2 T(s)-1$ pode ser vista em Schei (1994), e é feita usando a malha fechada, $T(s)$, sem abrir o laço de controle da Fig. 1. Note que a saída da malha fechada coincide com $y_{o}$ apenas no caso em que $F(s)=T(s)$.

\subsection{Procedimento Básico}

Dada uma função de tranferência $H(s)$, o procedimento anterior pode ser generalizado para obter ciclos limite controlados, em freqüências nas quais $H(s)$ possui um determinado ganho. Com isto, define-se o procedimento básico, apresentado pela proposição seguinte.

Proposição 2 Considere o sistema em malha fechada com relé mostrado na Fig. 3. Assuma que, para uma função de transferência estável $H(s)$ e um número real positivo $r$, a função de transferência

$$
F(s, r)=\frac{H(s)-r}{H(s)+r}
$$

também é estável. Então, se existe um ciclo limite, ele ocorre em uma freqüência $\omega_{0}$ tal que

$$
\left|H\left(j \omega_{o}\right)\right| \cong r .
$$

Prova: Se o sistema em malha fechada com relé apresenta um ciclo limite, então da análise por função descritiva tem-se que, na freqüência $\omega_{o}, \angle F\left(j \omega_{o}, r\right) \cong-90^{0}$. Então

$$
F\left(j \omega_{o}, r\right)=\frac{H\left(j \omega_{o}\right)-r}{H\left(j \omega_{o}\right)+r} \cong-k j,
$$

para algum $k>0$. Novamente,

$$
H\left(j \omega_{o}\right) \cong r \frac{1-k j}{1+k j},
$$

de modo que

$$
\left|H\left(j \omega_{o}\right)\right| \cong r
$$

Este é um resultado geral, uma vez que a função $H(s)$ pode assumir várias formas diferentes, inclusive a utilizada em Schei (1994), em que $H(s)=L(s)$ e $r=1$.

\section{ESTIMAÇÃO DA FUNÇÃO DE SENSI- BILIDADE}

A estimação da Função de Sensibilidade a partir do procedimento básico é apresentada na seqüência. 
Proposição 3 Considere o sistema em malha fechada com relé mostrado na Fig. 3. Assuma que, para uma Função de Sensibilidade Complementar estável $T(s)$ e um número real positivo $r$, a função de transferência

$$
F(s, r)=2 \frac{\frac{1}{r+1}}{1-\frac{r}{r+1} T(s)}-1,
$$

também é estável. Então, se há um ciclo limite, a freqüência de oscilação $\omega_{o}$ é tal que

$$
\left|S\left(j \omega_{o}\right)\right| \cong \frac{1}{r} .
$$

Prova: Como

$$
T(s)=\frac{L(s)}{1+L(s)}
$$

então,

$$
\begin{aligned}
F(s, r) & =\frac{2}{r} \frac{\frac{r}{r+1}}{1-\frac{r}{r+1} \frac{L(s)}{1+L(s)}}-1 \\
& =\frac{2(1+L(s))}{(r+1)(1+L(s))-r L(s)}-1 \\
& =\frac{2(1+L(s))}{1+L(s)+r}-1=\frac{1+L(s)-r}{1+L(s)+r} .
\end{aligned}
$$

Fazendo $H(s)=1+L(s)$ na Proposição (2), tem-se que

$$
\left|1+L\left(j \omega_{o}\right)\right| \cong r,
$$

ou, de maneira equivalente,

$$
\left|S\left(j \omega_{o}\right)\right|=\frac{1}{\left|1+L\left(j \omega_{o}\right)\right|} \approx \frac{1}{r} .
$$

O sistema em malha fechada que leva ao procedimento descrito é apresentado na Fig. 4.

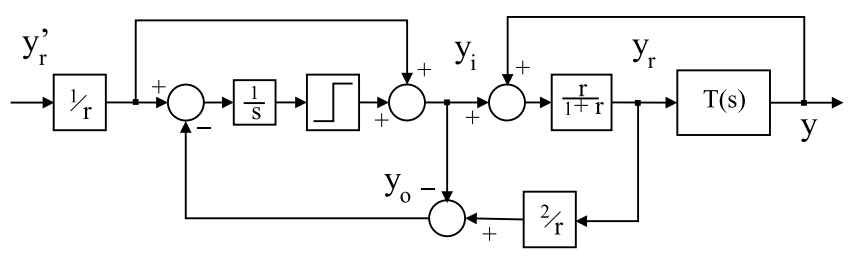

Figura 4: Diagrama em Blocos para Identificação da Função de Sensibilidade.

\section{DISCUSSÃO SOBRE CONDIÇÕES EX- PERIMENTAIS}

O experimento original do método do relé é realizado sob condições de operação bem definidas (Åström e Hägglund, 1984). A amplitude da oscilação na saída do processo pode ser controlada diretamente pela amplitude do relé. Além disso, se o processo é estável, então a malha fechada com relé leva, na maioria das vezes, a uma oscilação estável.

Nesta seção, são considerados detalhes das condições de operação, como a escolha da amplitude do relé, a estabilidade do experimento de identificação da Função de Sensibilidade e limites na amplitude dos sinais durante o experimento.

\subsection{Amplitude do Relé}

Considere o sistema em malha fechada mostrado na Fig. 4. O sinal $y_{i}(t)$ pode ser calculado como a contribuição de dois termos: o sinal de referência $y_{r}^{\prime}(t)$ ponderado por $1 / r$ e a saída do relé, $\pm d$, i.e.,

$$
y_{i}(t)=\frac{y_{r}^{\prime}(t)}{r} \pm d
$$

Assuma um sinal de referência $y_{r}^{\prime}$ constante, $T(s)$ estável e $T(0)=1$. Se a amplitude do relé é nula, i.e, $d=0$, então a função de transferência de $y_{i}(t)$ para $y(t)$ na Fig. 4 é dada por

$$
\frac{Y(s)}{Y_{i}(s)}=\frac{r T(s)}{1+r-r T(s)} .
$$

Em regime estacionário, tem-se que

$$
y=\frac{r}{r+1-r} y_{i}=r y_{i}=y_{r}^{\prime} .
$$

Conside agora que $y_{r}^{\prime}(t)=0$ e suponha que o sistema em malha fechada oscila na frequiência $\omega_{o}$. A amplitude $Y_{1}$ do primeiro harmônico do sinal de saída do processo é dada por

$$
Y_{1}=\frac{\left|r T\left(j \omega_{o}\right)\right|}{\left|1+r-r T\left(j \omega_{o}\right)\right|} Y_{i_{1}}
$$

onde $Y_{i_{1}}$ é a amplitude do primeiro harmônico do sinal periódico de entrada $y_{i}(t)$. Como a saída do relé é simétrica e de amplitude $d$, o sinal periódico $y_{i}(t)$ é um sinal quadrado simétrico de amplitude $d$. Assim, a amplitude do primeiro harmônico de $y_{i}(t)$ é dada por (Åström e Hägglund, 1995)

$$
Y_{i_{1}}=\frac{4 d}{\pi} \text {. }
$$


Sob algumas hipóteses, é possível obter um limite para $Y_{1}$, i.e., a amplitude da oscilação na saída do processo. Usando a desigualdade triangular na Eq. (6), tem-se

$$
\begin{aligned}
Y_{1}=\frac{\left|r T\left(j \omega_{o}\right)\right|}{\left|1+r-r T\left(j \omega_{o}\right)\right|} \frac{4 d}{\pi} & \leq \frac{\left|r T\left(j \omega_{o}\right)\right|}{|1+r|-\left|r T\left(j \omega_{o}\right)\right|} \frac{4 d}{\pi} \\
& =\frac{r\left|T\left(j \omega_{o}\right)\right|}{1+r-r\left|T\left(j \omega_{o}\right)\right|} \frac{4 d}{\pi} .
\end{aligned}
$$

Se $\left|T\left(j \omega_{o}\right)\right|=1$ e $r>1$, então a seguinte desigualdade é válida,

$$
Y_{1} \leq \frac{4 d}{\pi} r
$$

Esta informação pode ser utilizada para manter a saída $y(t)$ entre níveis de operação desejados. Por exemplo, supondo que $\Delta y_{\max }$ é a variação máxima admitida na saída do processo, ou seja, $y_{r}^{\prime}-\Delta y_{\max } \leq y(t) \leq$ $y_{r}^{\prime}+\Delta y_{\max }$, pode-se definir a amplitude do primeiro harmônico $Y_{1}$ como sendo,

$$
Y_{1} \leq \Delta y_{\max }=\frac{4 d}{\pi} r
$$

de modo que a amplitude do relé deve ser ponderada por $1 / r$, para que a amplitude de oscilação da saída do processo se mantenha no mesmo nível para diferentes valores de $r$. Então,

$$
d=\frac{\pi \Delta y_{\max }}{4} \cdot \frac{1}{r} .
$$

\subsection{Estabilidade e Limites de Sinais}

A estabilidade do experimento proposto é uma necessidade prática e deve ser garantida, para viabilizar sua utilização em identificação de sistemas.

Considere o sistema em malha fechada com relé apresentado na Fig. 3. Se o sinal $y_{r}^{\prime}(t)$ é limitado e a amplitude do relé é $d$, então $y_{i}(t)$ é um sinal limitado por construção. Como $Y_{o}(s)=F(s, r) Y_{i}(s)$, então $y_{o}(t)$ é limitado se $F(s, r)$ é estável para algum $r$. Considere agora o procedimento básico com $F(s, r)$ implementado conforme a Eq. (3). A estabilidade da estrutura em malha fechada pode ser avaliada da seguinte maneira.

Proposição $4 \mathrm{O}$ conjunto de números reais $r$ para os quais $F(s, r)$ é estável pode ser obtido diretamente do lugar de raízes de $H(s)$.

Prova: Considere

$$
H(s)=\frac{N(s)}{D(s)} .
$$

Então

$$
\begin{array}{r}
F(s, r)=\frac{H(s)-r}{H(s)+r}=\frac{N(s)-r D(s)}{N(s)+r D(s)} \\
=\frac{\frac{1}{r} N(s)-D(s)}{\frac{1}{r} N(s)+D(s)},
\end{array}
$$

de modo que a equação característica da malha fechada é dada pelo lugar de raízes de $H(s)$, com ganho de realimentação dado por $k=1 / r$.

Comentário 5 Em Schei (1994) $H(s)=L(s)$ e $r=1$ de modo que a função $F(s, r)$ resultante é estável por construção.

Do resultado anterior, a malha fechada da estrutura na Fig. 4 pode ser instável, dependendo de $H(s)$ e $r$. Em situações práticas, deve-se garantir que o sinal de saída do processo seja limitado, e que, além disso, esteja entre limites bem definidos.

Para o experimento de identificação da Função de Sensibilidade mostrado na Fig. 4, a estabilidade depende somente do laço de realimentação mostrado na Fig. 5. Se $T(s)$ é estável e $T(0)=1$, então para prevenir sinais não limitados na saída do processo, pode ser utilizada uma não linearidade do tipo saturação antes de $T(s)$, conforme mostrado na Fig. 6.

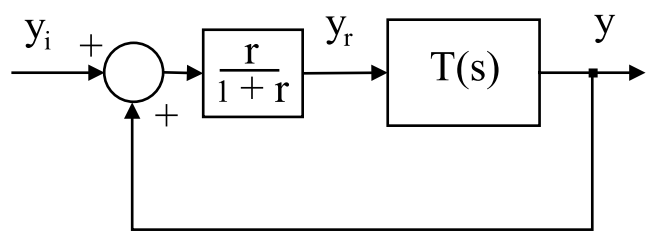

Figura 5: Estabilidade do Experimento com Relé em Malha Fechada.

Assim, dada a variação desejada na saída do processo, ou seja, $y_{r}^{\prime}-\Delta y_{\max } \leq y(t) \leq y_{r}^{\prime}+\Delta y_{\max }$, a não linearidade tipo saturação pode ser escolhida como

$$
y_{r}(t)=\left\{\begin{array}{l}
x(t), \text { se } \\
y_{r}^{\prime}(t)-\Delta y_{\max }<x(t)<y_{r}^{\prime}(t)+\Delta y_{\max }, \\
y_{r}^{\prime}(t)-\Delta y_{\max }, \text { se } x(t) \leq y_{r}^{\prime}(t)-\Delta y_{\max } \\
y_{r}^{\prime}(t)+\Delta y_{\max } \text { se } x(t) \geq y_{r}^{\prime}(t)+\Delta y_{\max }
\end{array}\right.
$$

Durante o experimento, pode-se variar $r$ até que a região de saturação seja alcançada. Quando isto ocorre, o experimento deve ser interrompido, pois o limite de estabilidade foi alcançado. 


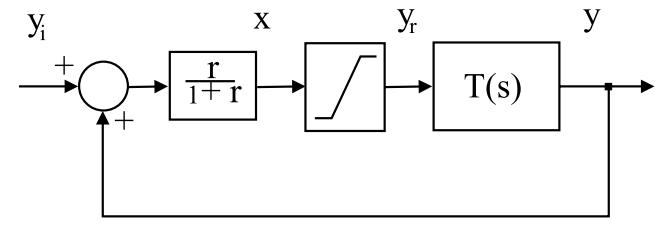

Figura 6: Experimento com Relé com Saturação na Entrada do Processo.

\section{ESTIMAÇÃO DA RESPOSTA EM FREQÜÊNCIA}

A análise por função descritiva aplicada a um sistema em malha fechada com relé usa a hipótese de que apenas o primeiro harmônico está presente na saída do processo, conforme descrito em Åström e Hägglund (1984). Quando a presença de harmônicos de ordem elevada não pode ser desprezada, a aproximação se torna pior, à medida que se aumenta a amplitude dos harmônicos superiores no sinal de saída. Se o relé é utilizado na estimação do ganho e freqüência críticos (Åström e Hägglund, 1984), o erro devido à analise por função descritiva é relativamente pequeno, pois, nestes casos, o ciclo limite normalmente ocorre em uma freqüência mais alta do que a freqüência de cruzamento do ganho. No entanto, quando harmônicas de ordem superior estão presentes, a aproximação se torna pior à medida em que a influência das demais harmônicas se torna mais significativa.

Uma maneira de contornar esta situação é utilizar a abordagem da Estimação da Função de Transferência Empírica (Empirical Transfer Function Estimation ETFE, Ljung (1999)). A análise de Fourier aplicada a sinais discretos é utilizada para estimar o ponto da curva de Nyquist (ganho e fase) correspondente à freqüência fundamental, bem como para harmônicos de ordem superior.

Considere $N$ pontos, $N$ par, do sinal $y(t)$ amostrado com período $T_{s}$. Defina $Y(\omega)$ como sendo

$$
Y(\omega)=\frac{1}{\sqrt{N}} \sum_{k=1}^{N} y(k) e^{-j \omega k} .
$$

Os valores de $Y(\omega)$ para $\omega=2 \pi n / N, n=1, \ldots, N$, correspondem à transformada discreta de Fourier (DFT) do sinal $y\left(k T_{s}\right)$, abreviado aqui por $y(k), 1<k<N$. Se $y(k)$ é periódico, ou seja, $y(k)=y\left(k+N_{o}\right)$, e $N=$ $s \cdot N_{o}$, para qualquer inteiro positivo $s$, então

$$
y(k)=\frac{1}{\sqrt{N_{o}}} \sum_{r=-N_{o} / 2+1}^{N_{o} / 2+1} A_{r} e^{2 \pi i k r / N_{o}}
$$

com

$$
A_{r}=\frac{1}{\sqrt{N_{o}}} \sum_{k=1}^{N_{o}} y(k) e^{-2 \pi i k r / N_{o}} .
$$

Além disso,

$\left|Y_{N}(\omega)\right|^{2}=\left\{\begin{array}{rl}s \cdot\left|A_{r}\right|^{2}, & \text { if } \omega=\frac{2 \pi r}{N_{o}}, r=0, \pm 1 \ldots \pm \frac{N_{o}}{2} \\ 0, & \text { if } \omega=\frac{2 \pi k}{N}, \quad k \neq r \cdot s\end{array}\right.$.

Este resultado segue de Ljung (1999). Considere agora a seguinte estimativa da função de transferência,

$$
\hat{T}\left(e^{j \omega}\right)=\frac{Y_{N}(\omega)}{Y_{R_{N}}(\omega)} .
$$

De Ljung (1999), tem-se que a estimativa dada pela Eq.

(9) possui as seguintes propriedades:

1. $\hat{T}$ é definida apenas para um certo número de freqüências,

2. Nestas freqüências, a estimativa é não tendenciosa, e a variância decai com $1 / \sqrt{N}$.

A estimativa $\hat{T}\left(e^{j \omega}\right)$ pode ser utilizada diretamente para obter o valor correspondente da Função de Sensibilidade para o sistema da Fig. 1, a partir da Eq. (9) e notando que $S+T=1$, ou seja,

$$
\hat{S}\left(e^{j \omega}\right)=1-\hat{T}\left(e^{j \omega}\right) .
$$

A Eq.(10) pode então ser utilizada para obter estimativas mais precisas para a Função de Sensibilidade. Em particular, quando os experimentos com relé são realizados para valores de $r$ que levam a oscilações de baixa freqüência, é possível obter estimativas razoáveis para outros harmônicos, além da fundamental. Note que a estimação é feita com dados de entrada e saída do sistema em malha fechada.

\section{EXEMPLO DE SIMULAÇÃO}

Considere um processo dado por

$$
G(s)=\frac{1}{(s+1)^{3}},
$$

e um controlador PI com a seguinte função de transferência

$$
C(s)=\frac{K_{p}\left(s+1 / T_{i}\right)}{s}=3,2 \frac{s+0,3446}{s} .
$$

A Função de Sensibilidade da malha fechada é dada por

$$
S(s)=\frac{s(s+1)^{3}}{s^{4}+3 s^{3}+3 s^{2}+4,2 s+1,1027} .
$$




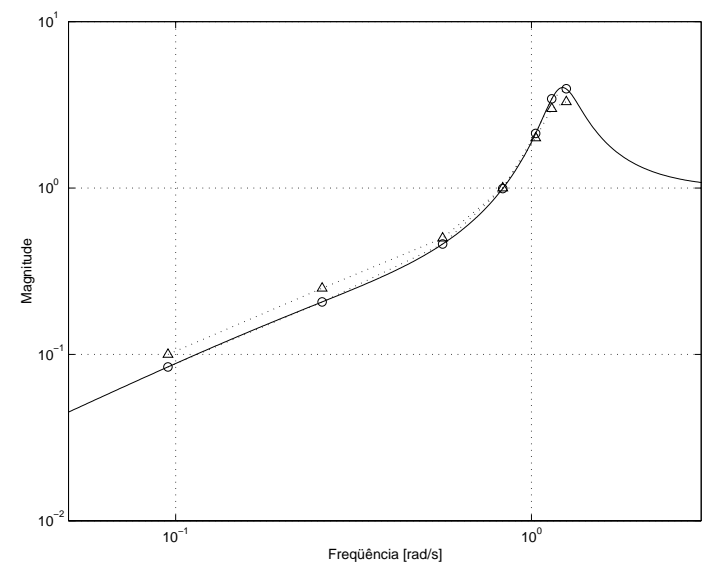

Figura 7: Estimação da Função de Sensibilidade: (-) curva real, $(\triangle)$ valor de $r^{-1}$ e (o) correção por DFT.

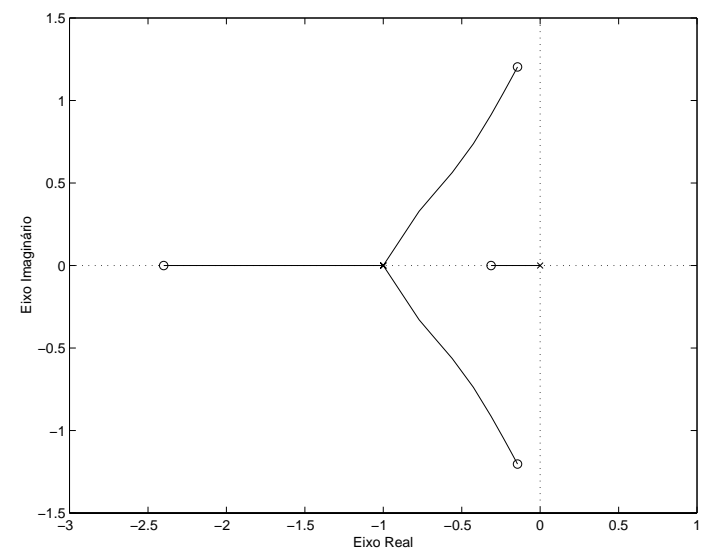

Figura 8: Lugar de Raízes de $H(s)=1+L(s)$.

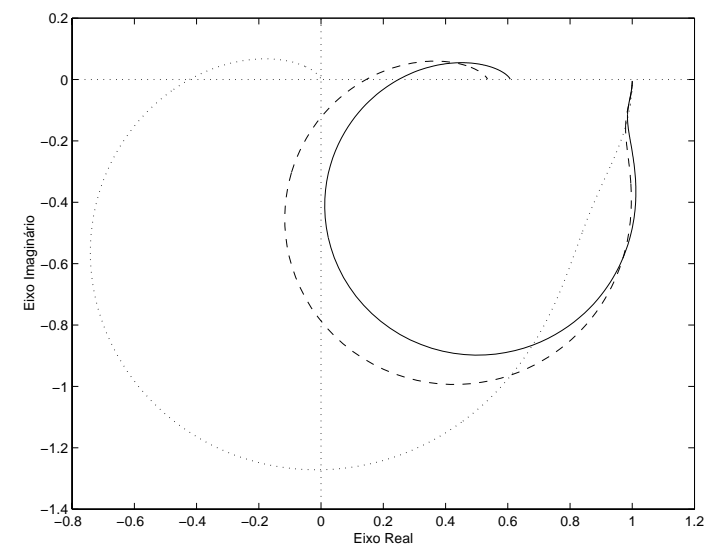

Figura 9: Curvas de Nyquist de $F(j \omega, r) .(\cdot) r^{-1}=1,0$ $,(--) r^{-1}=3,0$ e $(-) r^{-1}=4,1$.
O experimento em malha fechada para estimação da Função de Sensibilidade é executado conforme mostrado na Fig. 4, incluindo o bloco de saturação, conforme ilustrado na Fig. 6. Os resultados de estimação são mostrados na Fig. 7, utilizando valores de $r^{-1}$ no conjunto $[0,1 ; 0,25 ; 0,5 ; 1,0 ; 2,0 ; 3,0 ; 3,3]$. Para avaliação dos resultados, também é mostrada na mesma figura a curva real de magnitude, a partir da Eq. 11.

Neste caso, não foi observada instabilidade no experimento para nenhum valor de $r^{-1}$ do conjunto especificado. De fato, pode-se observar do lugar de raízes de $H(s)=1+L(s)$, mostrado na Fig. 8, que o experimento é estável para qualquer $r^{-1}>0$.

O valor máximo da magnitude da Função de Sensibilidade foi obtido reduzindo o ganho $r$ (i.e, aumentando $r^{-1}$ ) até que o ciclo limite fosse interrompido. Curvas de Nyquist de $F(r, j \omega)$ para alguns valores de $r$ são mostradas na Fig. 9. Note que para para $r^{-1}>4,1$, a curva de Nyquist de $F(r, j \omega)$ não intercepta o semi-eixo imaginário negativo o que indica a inexistência de ciclos limite devido ao relé para tais valores de ganho.

As saídas do processo para $r^{-1}=0,1, r^{-1}=3,0 \mathrm{e}$ $r^{-1}=4,0$ são mostradas na Fig. 10. Para este exemplo, o ciclo limite desaparece para $r^{-1} \geq 3,3$, e uma estimativa do pico da Função de Sensibilidade pode ser obtida a partir do valor corrigido por DFT, de modo que

$$
\max _{\omega}|\hat{S}(j \omega)|=3,95 .
$$

O valor real para o pico da Função de Sensibilidade do exemplo é aproximadamente

$$
\max _{\omega}|S(j \omega)| \cong 4,0187 .
$$

\section{RESULTADOS EXPERIMENTAIS}

Nesta seção, são apresentados resultados experimentais obtidos com um modelo reduzido de um sistema térmico (trocador de calor). Um diagrama em blocos do sistema é mostrado na Fig. 11.

A energia térmica é fornecida ao sistema pela resistência elétrica $R$. A tensão elétrica aplicada à resistência é obtida através de um retificador com ângulo de disparo controlado por um sinal de tensão de 0 a $2,5 \mathrm{~V}$, conforme ilustrado na figura. $\mathrm{O}$ ventilador $V_{1}$ cria um fluxo de ar da entrada para o interior do sistema passando pela resistência $R$. $\mathrm{O}$ ventilador $V_{2}$ introduz ar à temperatura ambiente no sistema, para avaliar efeitos de perturbação. A temperatura é medida pelo sensor térmico $S_{1}$ na forma de um sinal de tensão de 0 a $2,5 \mathrm{~V}$. O controlador 


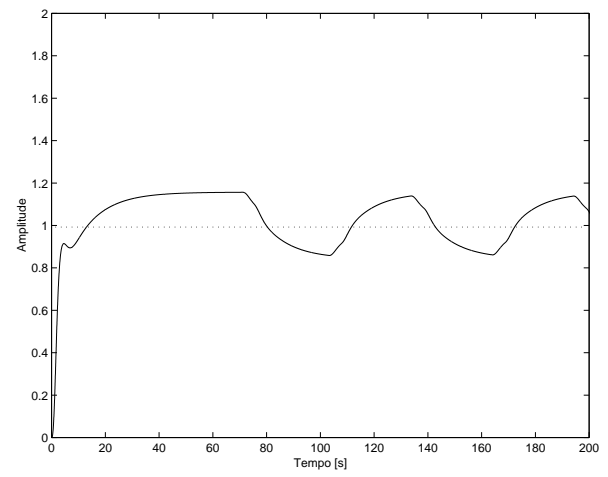

(a) $r^{-1}=0,1$.

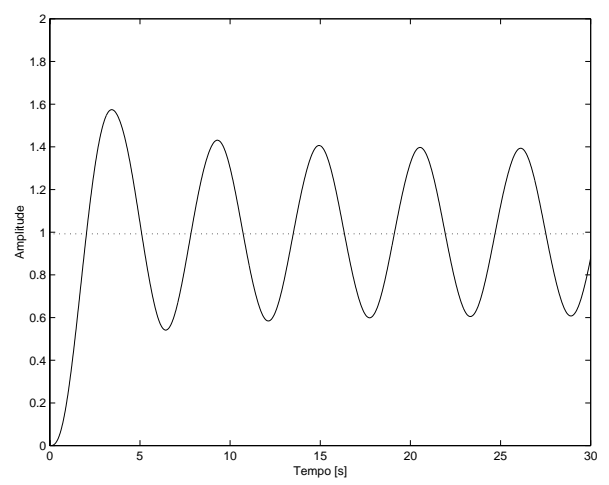

(b) $r^{-1}=3,0$.

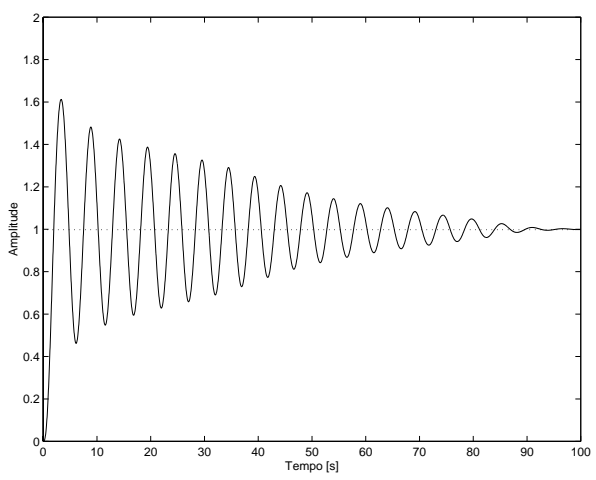

(c) $r^{-1}=4,0$.

Figura 10: Saída da malha fechada do exemplo de simulação para alguns valores de $r$.

é implementado em um microcomputador com sistema de aquisição de dados (conversores Analógico/Digital e Digital/Analógico), não mostrado na figura. O objetivo

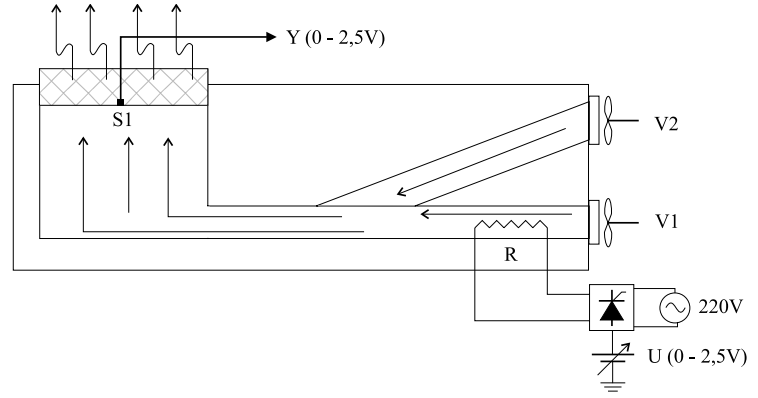

Figura 11: Diagrama em Blocos do Sistema Térmico Utilizado no Experimento.

é manter a temperatura constante no ponto de medição.

Escolheu-se como ponto de operação do sistema o valor de $y_{r}=1,25 \mathrm{~V}$. O método do relé aplicado ao processo em torno do ponto de operação escolhido é mostrado na Fig. 13. Os valores de ganho e período críticos obtidos do experimento levam ao controlador $C_{1}(s)$ obtido pela tabela de Ziegler e Nichols (ver Åström e Hägglund (1995)),

$$
C_{1}(s)=\frac{K_{p 1}\left(s+1 / T_{i 1}\right)}{s}=2,90 \frac{(s+0,1848)}{s}
$$

com

$$
K_{p}=2,90 \text { e } T_{i}=5,41 .
$$

Tabela 1: Resultados para o controlador $C_{1}$.

\begin{tabular}{ccc}
\hline \hline$r^{-1}$ & $\left|\hat{S}\left(e^{j \omega}\right)\right|$ & $\omega$ \\
\hline \hline 0,5 & 0,466 & 0,133 \\
1,0 & 0,923 & 0,325 \\
1,5 & 1,416 & 0,492 \\
1,75 & 1,678 & 0,598 \\
$\mathbf{1 , 9}$ & $\mathbf{1 , 8 4 5}$ & $\mathbf{0 , 7 2 6}$ \\
\hline \hline
\end{tabular}

Tabela 2: Resultados para o controlador $C_{2}$.

\begin{tabular}{ccc}
\hline \hline$r^{-1}$ & $\left|\hat{S}\left(e^{j \omega}\right)\right|$ & $\omega$ \\
\hline \hline 0,5 & 0,437 & 0,193 \\
1,0 & 0,913 & 0,390 \\
1,5 & 1,408 & 0,500 \\
2,0 & 1,902 & 0,596 \\
$\mathbf{2 , 8}$ & $\mathbf{2 , 8 5 1}$ & $\mathbf{0 , 7 8 6}$ \\
\hline \hline
\end{tabular}

O experimento de identificação da Função de Sensibilidade é então aplicado à malha fechada resultante. A estrutura mostrada na Fig. 4 é usada em conjunto com o bloco de saturação mostrado na Fig. 6 . Os resultados são mostrados na Fig. 14, juntamente 


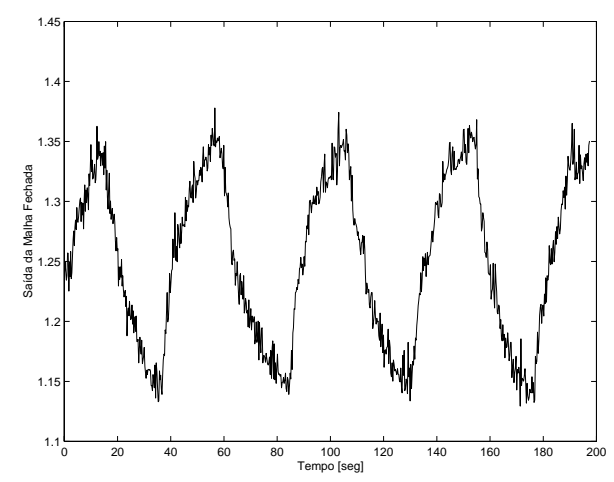

(a) $r^{-1}=0,5$.

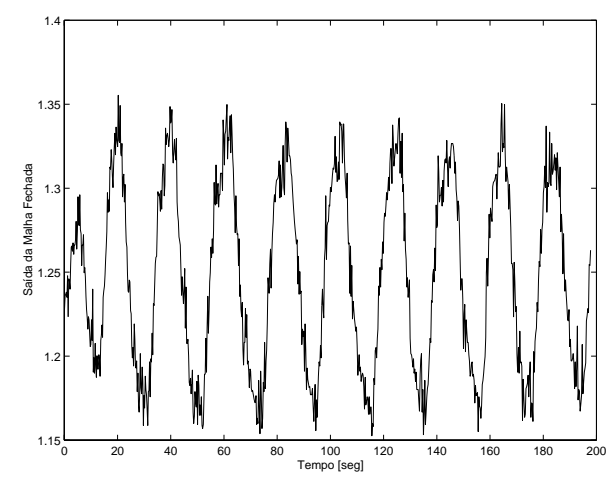

(b) $r^{-1}=1,0$.

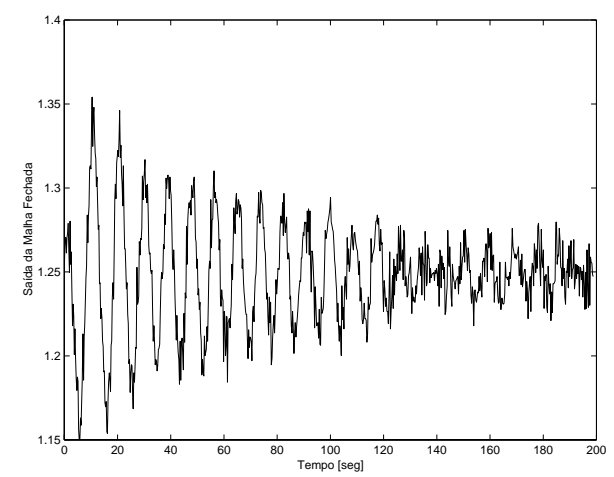

(c) $r^{-1}=2,0$.

Figura 12: Saída da malha fechada com o controlador $C_{1}(s)$ para alguns valores de $r$.

com os resultados obtidos para um segundo controlador, $C_{2}(s)$, com um aumento de $50 \%$ do ganho proporcional

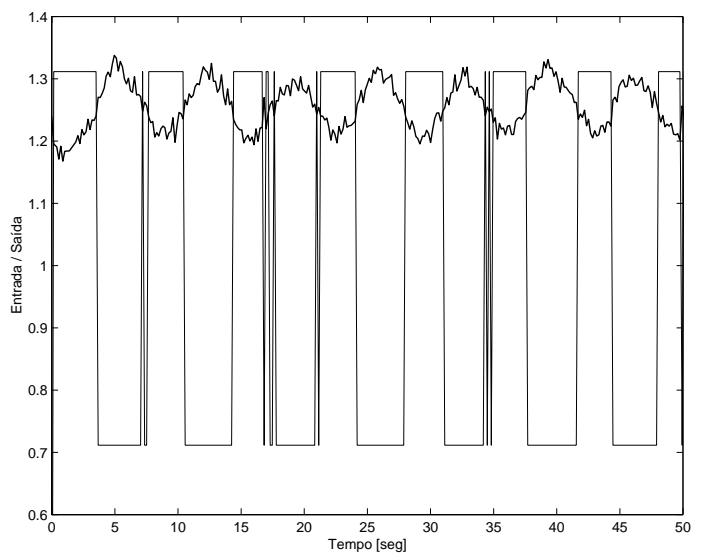

Figura 13: Entrada e Saída do processo durante o experimento com o método do relé.

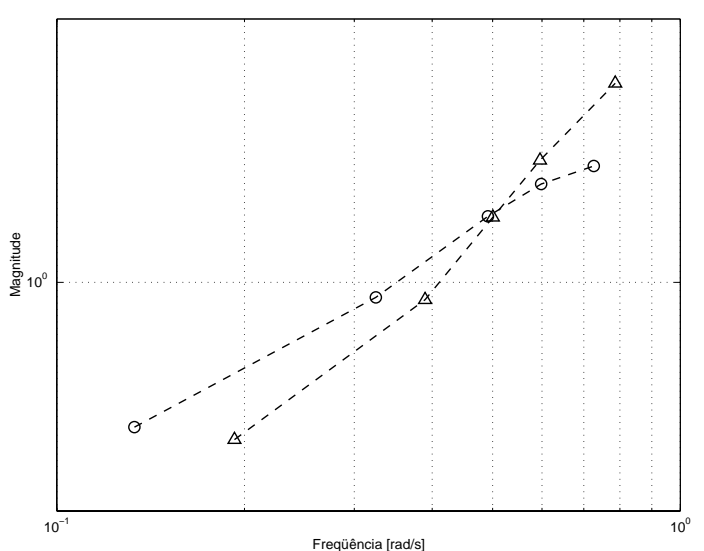

Figura 14: Resultados do Experimento de Identificação da Função de Sensibilidade: (o) controlador 1 e $(\triangle)$ controlador 2 .

$$
\begin{aligned}
& \left(K_{p 2}=1.5 K_{p 1} \text { e } T_{i 2}=T_{i 1}\right), \text { dado por } \\
& \quad C_{2}(s)=\frac{K_{p 2}\left(s+1 / T_{i 2}\right)}{s}=4,35 \frac{(s+0,1848)}{s}
\end{aligned}
$$

A saída da malha fechada durante o experimento, usando o controlador $C_{1}(s)$ para alguns valores de $r$ é mostrada na Fig. 12. Nas tabelas 1 e 2 são mostrados os valores obtidos para cada experimento realizado. O pico da função de sensibilidade é obtido como o maior valor de $r^{-1}$ tal que a oscilação na saída permanece, e é destacado em negrito nas tabelas para ambos controladores. 


\section{CONCLUSÃO}

Neste artigo, uma nova técnica de estimação da Função de Sensibilidade é apresentada. O procedimento é realizado com o sistema em malha fechada, em condições normais de operação. Aspectos práticos relativos à amplitude de oscilação na saída do processo são discutidos, e propõe-se a utilização de não-linearidades do tipo saturação na entrada do sistema em malha fechada. O experimento pode ser utilizado para análise de robustez de sistemas de controle, ou sintonia de controladores com especificações na Função de Sensibilidade. Resultados experimentais obtidos com um sistema de controle de temperatura em escala de laboratório são apresentados para ilustração da técnica de identificação.

\section{REFERÊNCIAS}

Åström, K. J. e Hägglund, T. (1984). Automatic tuning of simple regulators with specifications on phase and amplitude margins, Automatica 20(5): 645651.

Åström, K. J. e Hägglund, T. (1995). PID Controllers: Theory, Design and Tuning, 2nd edn, Instrument Society of America, Research Triangle Park, North Carolina.

Barros, P. R. e Wittenmark, B. (1997). Frequency domain sensitivity shaping using overparametrized controllers, 34rd IEEE CDC - Conference on Decision and Control, San Diego - CA, USA, pp. 27162721.

de Arruda, G. H. M. e Barros, P. R. (2000a). Estimação da função de sensibilidade baseada em experimento com relé em malha fechada, XIII CBA, Congresso Brasileiro de Automática, Florianópolis, PR, pp. 2004-2009.

de Arruda, G. H. M. e Barros, P. R. (2000b). Moldagem da função de malha baseada no método do relé em malha fechada, XIII CBA, Congresso Brasileiro de Automática, Florianópolis, PR, pp. 1998-2003.

Doyle, J. C., Francis, B. A. e Tannenbaum, A. R. (1995). Feedback Control Theory, Macmillan Publishing Company, Inc., Englewood Cliffs, NJ.

Langer, J. e Landau, I. D. (1999). Combined pole Placement/Sensitivity function shaping method using convex optimization criteria, Automatica 35(6): 1111-1120.

Ljung, L. (1999). System Identification: Theory for the User, 2nd edn, Prentice Hall, Upper Saddle River, NJ.
Schei, T. S. (1992). A method for closed loop automatic tuning of PID controllers, Automatica 28(3): 587591.

Schei, T. S. (1994). Automatic tuning of PID controllers based on transfer function estimation, Automatica 30(12): 1983-1989.

Tan, K. K., Wang, Q.-G., Hang, C. C. e Hägglund, T. J. (1999). Advances in PID Control, Springer-Verlag, London.

Wang, L., Desarmo, M. L. e Cluett, W. R. (1999). Realtime estimation of process frequency response and step response from relay feedback experiments, $A u$ tomatica 35(8): 1427-1436.

Wang, Q.-G., Hang, C.-C. e Bi, Q. (1999). A technique for frequency response identification from relay feedback, IEEE Transactions on Control Systems Technology 7(1): 122-128.

Ziegler, J. G. e Nichols, N. B. (1942). Optimum settings for automatic controllers, Transactions of the ASME 42: 759-768. 